\title{
Mortality and glycemic control among patients with acute and chronic myeloid leukemia and diabetes: a case-control study
}

\author{
Julia E Wiedmeier ${ }^{1}$ (D), Luke J Mountjoy ${ }^{2,3}$ (D), Matthew R Buras ${ }^{4}$ (D) Heidi E Kosiorek ${ }^{4}$ (D) \\ Kyle E Coppola ${ }^{5}$ (D) Patricia M Verona ${ }^{6}$ (D) , Curtiss B $\operatorname{Cook}^{7}$ (D) \& Nina J Karlin*,2,5 (D) \\ ${ }^{1}$ Department of Internal Medicine, Mayo Clinic Hospital, 5777 E Mayo Blvd, Phoenix, AZ 85054, USA \\ ${ }^{2}$ Division of Hematology \& Medical Oncology, Mayo Clinic Hospital, 5777 E Mayo Blvd, Phoenix, AZ 85054, USA \\ ${ }^{3}$ Colorado Blood Cancer Institute, 1721 E 19th Ave, Suites 200-300, Denver, CO 80218, USA \\ ${ }^{4}$ Biostatistics, Mayo Clinic, 13400 E. SheaBlvd., Scottsdale, AZ 85259, USA \\ ${ }^{5}$ Mayo Clinic Cancer Center, Mayo Clinic, 13400 E. Shea Blvd., Scottsdale, AZ 85259, USA \\ ${ }^{6}$ Enterprise Technology Services, Mayo Clinic Hospital, 5777 E Mayo Blvd, Phoenix, AZ 85054, USA \\ ${ }^{7}$ Division of Endocrinology, Mayo Clinic, 13400 E. Shea Blvd., Scottsdale, AZ 85259, USA \\ *Author for correspondence: karlin.nina@mayo.edu
}

\begin{abstract}
Aim: We examined the association between diabetes and survival in patients with acute and chronic myeloid leukemia and the association of leukemia with glycemic control. Patients \& methods: Patients with leukemia with and without diabetes (2007-2015) were retrospectively identified and matched 1:1 ( $n=70$ per group). Overall survival was estimated by the Kaplan-Meier method. Hemoglobin $A_{1 c}$ and glucose levels the year after leukemia diagnosis were compared by mixed models. Results: Among 25 of 70 patients with diabetes, mean hemoglobin $A_{1 c}$ during the year after leukemia diagnosis was $6.8 \%$. Kaplan-Meier-estimated 3-year survival was $46 \%$ for diabetes patients versus $45 \%$ for controls $(p=0.79)$. Conclusion: No associations were found between leukemia, diabetes, survival and glycemic control.

Lay abstract: The goal of this study was to evaluate the association between diabetes and survival among patients with acute and chronic myeloid leukemia and the association between leukemia and glycemic control. A total of 140 patients with acute and chronic leukemia were retrospectively identified (70 with diabetes; 70 without diabetes). In this study, diabetes was not associated with decreased survival in patients with leukemia and leukemia was not associated with poor glycemic control.
\end{abstract}

First draft submitted: 31 July 2020; Accepted for publication: 18 September 2020; Published online:

26 October 2020

Keywords: cancer $\bullet$ endocrinology • outcomes research

Leukemia is one of the ten most common causes of cancer deaths in the USA [1]. It has a higher incidence in White persons and males. A common comorbid condition in many people with and without cancer is diabetes, present in an estimated $9.4 \%$ of the US population or 30.3 million people, as of 2017 [2]. Studies have shown an increased risk of death of certain solid cancers in patients with diabetes [3,4], but data are limited and inconsistent regarding the association between leukemia, diabetes and death.

The presence of diabetes in patients with cancer could conceivably lead to worse cancer prognosis. There are several possible mechanisms for the association between cancer and diabetes. Diabetes is a state of insulin resistance leading to abnormal glucose utilization and disturbances of glucose metabolism, which leads to chronic hyperglycemia [5] and cancer cells consume more glucose than normal cells [6]. Increased levels of circulating glucose allows malignant cells more access to glucose and thus an environment that gives cancer cells the nutrients to thrive and grow. Patients with diabetes often have increased levels of circulating insulin, which may also increase growth and proliferation of cancer cells [7]. Obesity and diabetes are chronic states of inflammation, which has also been shown to promote tumor growth and survival [8]. 
A large prospective cohort study in the US evaluated diabetes as a predictor of cancer death and found a significant association with solid organ cancers, but there was no significant relationship in diabetes patients with leukemia [9]. Another prospective cohort study among South and East Asians did not show an increased risk of death in diabetes patients with leukemia [10]. A retrospective analysis found an increased 30-day mortality rate in patients with diabetes among older adults with acute myeloid leukemia (AML) receiving intensive therapy [11].

We have previously published case-control studies showing no association between diabetes and decreased survival in patients with melanoma, endometrial cancer, pancreatic cancer, colorectal cancer or lung cancer, but we did show an association between death and progression in patients with diabetes and esophageal and gastric cancer [12-17]. Given the paucity of studies examining the relationship between diabetes and hematologic cancers, we aimed to investigate whether the presence of diabetes was related to higher mortality rates in patients with leukemia. Moreover, few studies have evaluated for an association between leukemia and glycemic control among patients with diabetes. For this analysis, we focused on patients with AML and chronic myeloid leukemia (CML).

\section{Methods}

\section{Case selection}

Institutional review board approval was obtained for this retrospective case-control study. We searched our institutional cancer registry for the records of adult patients (>18 years) with newly diagnosed AML or CML who were seen between 1 January 2007 and 31 December 2015. Cancer cases were linked to the electronic health record to identify patients with a diagnosis of diabetes using the International Classification of Diseases, Ninth Revision diagnostic code 250.00, as previously described [12-16]. Electronic health record data were examined around the time of cancer diagnosis to identify patients who had diabetes claims during this period. Data were collected regarding age at leukemia diagnosis, diagnosis date, ethnicity, BMI, allelic variation/translocation status of leukemia and treatment. In addition, we collected data on marital status, tobacco use, alcohol use, payer type, Eastern Cooperative Oncology Group performance status, corticosteroid use and transplant status. We chose to evaluate myeloid leukemias because myeloid and lymphoid leukemias are different entities based on precursor cells and lymphoid leukemias warrant a separate study.

We then cross-referenced these data against a list of all patients seen during the same period who had a diagnosis of Type 1 or Type 2 diabetes to categorize patients with leukemia by diabetes status (with or without diabetes). Patients who had another primary cancer were excluded. From this dataset, patients with leukemia and diabetes were matched 1:1 via a greedy matching algorithm to control patients with leukemia without diabetes. Variables included in the matching algorithm were sex and age at leukemia diagnosis. Patients were excluded if no chart review was able to be conducted for their matched pair or if the patient's diabetes status could not be verified from the chart review.

Glucose and hemoglobin $\mathrm{A}_{1 \mathrm{c}}\left(\mathrm{HbA}_{1 \mathrm{c}}\right)$ values were derived from the laboratory information system. We then reviewed the electronic health record for additional detailed information on leukemia treatment (chemotherapy, targeted therapy, radiation therapy) and data related to diabetes (date of diabetes diagnosis, type of diabetic therapy and diabetic complications).

\section{Statistical analysis}

Patients with CML or AML with diabetes (cases) and without diabetes (controls) were compared on the basis of patient characteristics and clinical variables. Continuous variables were compared by using paired $t$ tests; categorical variables were compared by using the McNemar test or Bowker test for symmetry. $\mathrm{HbA}_{1 \mathrm{c}}$ levels during the first year after leukemia diagnosis were evaluated with a linear mixed model in the diabetes group only $\left(\mathrm{Hb} \mathrm{A}_{1 \mathrm{c}}\right.$ values were unavailable for most patients without diabetes). Time was considered a fixed effect, and an individual specific random effect was included. A similar approach was used for modeling glucose values during that year. Fixed effects included time in days, case or control designation, an interaction term (days $\times$ case-control designation) and patient-specific and matched pair-specific effects. Glycemic control was defined as a mean glucose value less than $126 \mathrm{mg} / \mathrm{dl}$ during the first year after cancer diagnosis.

Overall survival (OS) was defined as the time from leukemia diagnosis until death of any cause. For OS, patients were considered censored at the last known follow-up date if death was not documented in the health record. Three-year OS was estimated with the Kaplan-Meier method and compared between groups by using the log-rank test. Cox proportional hazards regression was used to assess for effect of diabetes and included OS, with matched pairs as the strata variable. Sample size was based on the number of available cases in 2007-2015; it provided 
Figure 1. Mean hemoglobin $A_{1 c}$ levels within 1 year after leukemia diagnosis in patients with diabetes $(\mathbf{n}=\mathbf{2 5})$. $\mathrm{HbA}_{1 \mathrm{c}}$ level did not change over time $(p=0.23)$.

$\mathrm{HbA}_{1 \mathrm{c}}$ : Hemoglobin $\mathrm{A}_{1 \mathrm{c}}$.

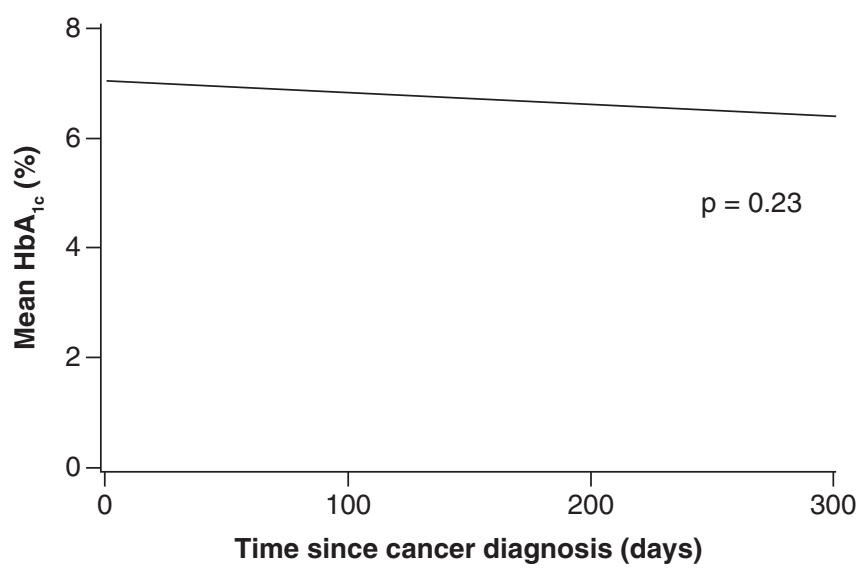

$80 \%$ power to detect a difference of $15 \%$ in 3-year survival rate estimates between cases and controls. p-value less than 0.05 was considered statistically significant; SAS version 9.4 (SAS Institute Inc., NC, USA) was used for analysis.

\section{Results}

\section{Patients with leukemia}

The original matched dataset contained 83 pairs; after exclusion of 13 pairs per the stated criteria, 70 matched pairs $(\mathrm{N}=140$ ) were included in the analysis (Table 1). The median age of patients at leukemia diagnosis was 62 (range: $19-94)$ years, $60 \%$ were men and $89 \%$ had AML. The majority of patients were White $(83.6 \%)$ and non-Hispanic (60.0\%) (Table 1).

Of AML and CML patients with data available, $79 \%$ of those with diabetes received corticosteroids, compared with $1 \%$ of those without diabetes $(\mathrm{p}<0.001)$, and the proportion with graft-versus-host disease was similar between patients with and without diabetes $(\mathrm{p}=0.52)$ (Table 1). Chemotherapy regimens were variable, with approximately 11 different conditioning regimens, at least 20 chemotherapy regimens and 15 different targeted therapy regimens (data not shown). Approximately $80 \%$ of patients with $(n=55)$ and without $(n=54)$ diabetes received some type of chemotherapy, whereas 11 patients without diabetes $(16 \%)$ and 15 patients with diabetes (21\%) had targeted therapy (data not shown). There was no significant difference in treatment regimens between patients with and without diabetes $(\mathrm{p}>0.99)$.

\section{Patients with diabetes}

\section{Glucose control \& treatment characteristics}

Among patients with diabetes, 25 had at least $1 \mathrm{HbA}_{1 \mathrm{c}}$ value measured within the year after leukemia diagnosis. For these patients, the mean (standard deviation) value was $6.8 \%(1.3 \%)$ and $\mathrm{HbA}_{1 \mathrm{c}}$ values did not change over time (Figure 1). Mean glucose values within the year after leukemia diagnosis were significantly different between patients with and without diabetes $(\mathrm{p}<0.001)$ (Figure 2). There was a significant interaction effect (days $\times$ diabetes group; $\mathrm{p}=0.01$ ); glucose values increased in the diabetes patients during the year after diagnosis but remained stable in those without diabetes (Figure 2). Among 46 patients with data available on diabetes treatment, the largest percentage was treated with oral medication alone $(n=20,43 \%)$, followed by insulin $(n=16,35 \%)$ and diet modification $(\mathrm{n}=7,15 \%)$.

\section{Diabetes effect on leukemia survival}

Median follow-up time was 23.2 months for surviving patients (Figure 3). The 3-year survival was estimated at 46\% (95\% CI: 35-61\%) for diabetes patients versus 45\% (95\% CI: 33-61\%) for patients without diabetes (p $=0.79$ ). The hazard ratio (stratified for matched pairs) was 1.05 (95\% CI: $0.57-1.94 ; \mathrm{p}=0.88$ ). The 3-year relapse-free survival was estimated at 34\% (95\% CI: $23-49 \%$ ) for diabetes patients versus $43 \%$ (95\% CI: 32-59\%) for patients without diabetes $(\mathrm{p}=0.58)$ (Figure 4). The hazard ratio (stratified for matched pairs) was $1.10(95 \%$ CI: 0.61-1.98; $\mathrm{p}=0.76)$. 
Table 1. Patient characteristics ${ }^{\dagger}$.

\begin{tabular}{|c|c|c|c|c|}
\hline \multirow[t]{2}{*}{ Characteristic ${ }^{\ddagger}$} & \multirow{2}{*}{ Total $(n=140)$} & \multicolumn{2}{|c|}{ Group } & \multirow[t]{2}{*}{ p-value } \\
\hline & & Diabetes $(n=70)$ & No diabetes $(n=70)$ & \\
\hline Age at diagnosis, y & $55.3(16.0)$ & $55.3(16.3)$ & $55.2(15.8)$ & NA \\
\hline Women & $56(40.0)$ & $28(40)$ & $28(40)$ & NA \\
\hline Ethnicity & & & & 0.98 \\
\hline Hispanic & $24(17.1)$ & $12(17)$ & $12(17)$ & \\
\hline Non-Hispanic & $84(60.0)$ & $43(61)$ & $41(59)$ & \\
\hline Unknown & $32(22.9)$ & $15(21)$ & $17(24)$ & \\
\hline Race & & & & 0.16 \\
\hline White & $117(83.6)$ & $57(81)$ & $60(86)$ & \\
\hline American Indian/Alaskan native & $6(4.3)$ & $6(9)$ & 0 & \\
\hline Asian & $5(3.6)$ & $1(1)$ & $4(6)$ & \\
\hline African-American & $4(2.9)$ & $2(3)$ & $2(3)$ & \\
\hline Other & $6(4.3)$ & $3(4)$ & $3(4)$ & \\
\hline Unknown & $2(1.4)$ & $1(1)$ & $1(1)$ & \\
\hline Leukemia type & & & & 0.60 \\
\hline Chronic & $16(11.4)$ & $9(13)$ & $7(10)$ & \\
\hline Acute & $124(88.6)$ & $61(87)$ & $63(90)$ & \\
\hline Acute leukemia subtype & $(n=96)$ & $(n=36)$ & $(n=60)$ & 0.01 \\
\hline Favorable & $14(14.6)$ & $5(14)$ & $9(15)$ & \\
\hline Intermediate & $33(34.4)$ & $6(17)$ & $27(45)$ & \\
\hline Unfavorable & $49(51.0)$ & $25(69)$ & $24(40)$ & \\
\hline Variation/translocation ${ }^{\S}$ & $(n=113)$ & $(n=52)$ & $(n=61)$ & 0.001 \\
\hline None & $43(38.1)$ & $10(19)$ & $33(54)$ & \\
\hline Translocation & $40(35.4)$ & $26(50)$ & $14(23)$ & \\
\hline Variation & $30(26.5)$ & $16(31)$ & $14(23)$ & \\
\hline Transplant & $67(51.5)(n=130)$ & $38(57)(n=67)$ & $29(46)(n=63)$ & 0.22 \\
\hline GVHD & $52(78.8)(n=66)$ & $31(82)(n=38)$ & $21(75)(n=28)$ & 0.52 \\
\hline BMI category & & & & 0.28 \\
\hline$<25.0$ & $51(36.4)$ & $21(30)$ & $30(43)$ & \\
\hline $25.0-29.9$ & $27(19.3)$ & $15(21)$ & $12(17)$ & \\
\hline$\geq 30.0$ & $62(44.3)$ & $34(49)$ & $28(40)$ & \\
\hline Any alcohol use & & & & 0.59 \\
\hline Yes & $51(36.4)$ & $23(33)$ & $28(40)$ & \\
\hline No & $84(60.0)$ & $44(63)$ & $40(57)$ & \\
\hline Unknown & $5(3.6)$ & $3(4)$ & $2(3)$ & \\
\hline Smoking status & & & & 0.74 \\
\hline Never & $70(50.0)$ & $36(51)$ & $34(49)$ & \\
\hline Former & $56(40.0)$ & $28(40)$ & $28(40)$ & \\
\hline Current & $9(6.4)$ & $3(4)$ & $6(9)$ & \\
\hline Unknown & $5(3.6)$ & $3(4)$ & $2(3)$ & \\
\hline Use of corticosteroids & $45(36.0)(n=125)$ & $44(79)(n=56)$ & $1(1)(n=69)$ & $<0.001$ \\
\hline
\end{tabular}

Diabetes effect on leukemia subtype in CML \& $A M L$

The majority of patients had AML (88.6\%) (Table 1). Among the 16 patients with CML, the vast majority were in the chronic phase (bone marrow and peripheral blood contain $<10 \%$ blasts, compared with blast phase in which bone and blood contain $>20 \%$ blasts) (data not shown). Allelic variation status was also compared in patients 


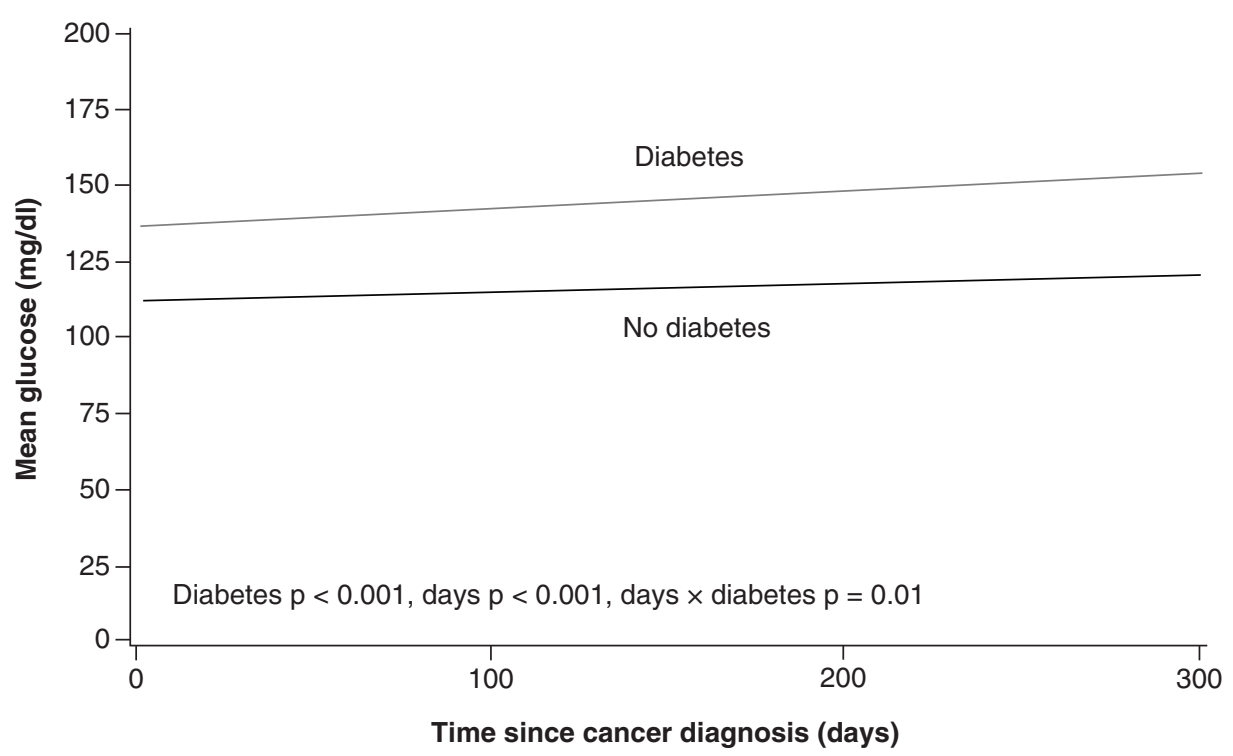

Figure 2. Mean glucose levels within 1 year after leukemia diagnosis for both groups. At the end of 1 year, mean glucose level was significantly higher in the diabetes group, but the mean glucose levels for both groups remained less than $180 \mathrm{mg} / \mathrm{dl}$ (fixed effects: diabetes, $\mathrm{p}<0.001$; days, $\mathrm{p}<0.001$; days $\times$ diabetes, $\mathrm{p}=0.01$ ).

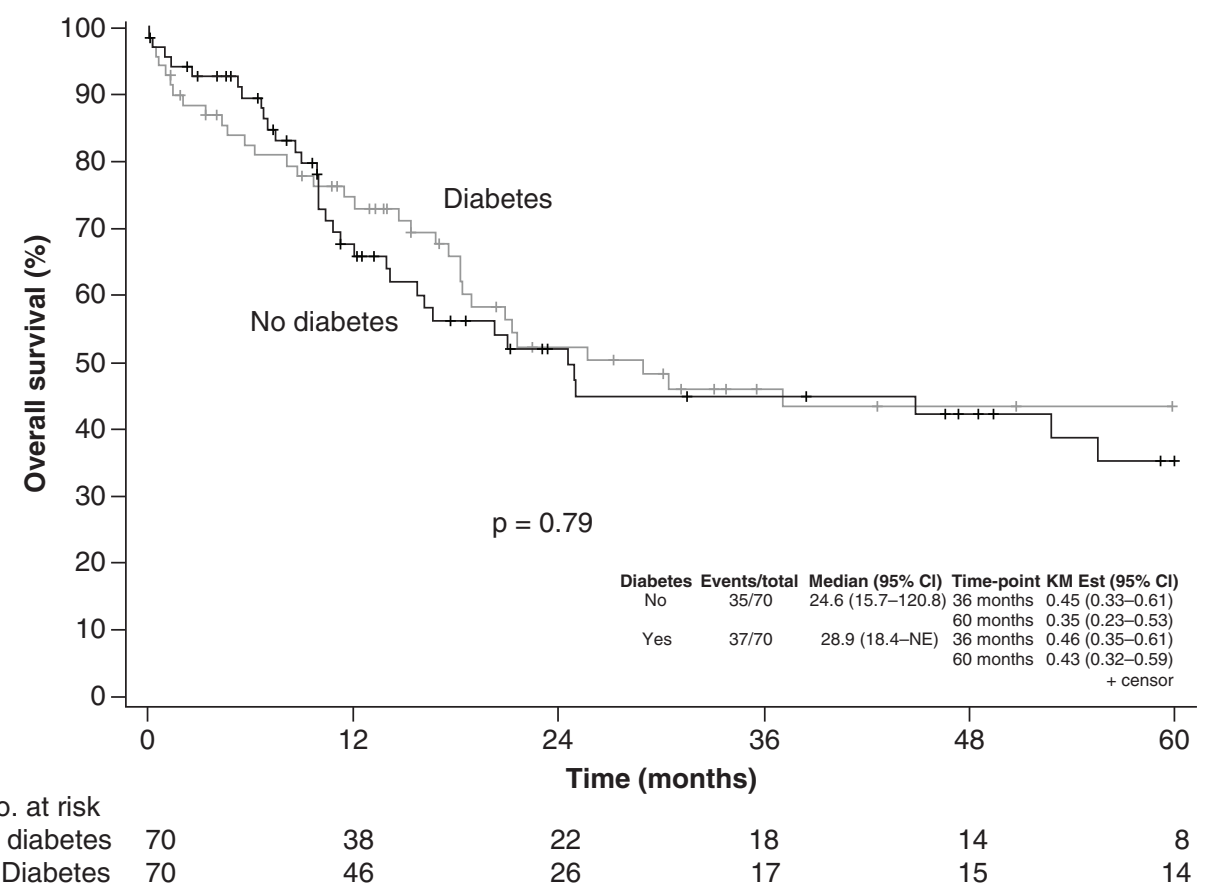

Figure 3. Kaplan-Meier curves estimating overall survival among patients with leukemia. There was no difference in survival between patients with and without diabetes $(p=0.79)$.

KM: Kaplan-Meier estimate; NE: Not estimated.

with AML. The percentage of patients with variations and/or translocations was significantly higher in the AML group with versus without diabetes (81 vs $46 \% ; \mathrm{p}=0.001$ ), although more patient data were missing among diabetes patients. For AML cases risk-stratified on the basis of cytogenetic and molecular features into favorable, intermediate and poor subtypes, patients with diabetes had a lower prevalence of the intermediate subtype (17 vs $45 \%$ for no diabetes) and a higher prevalence of the unfavorable subtype (69 vs $40 \%$ for no diabetes; $\mathrm{p}=0.01$ ). Again, more patient data were missing among diabetes patients. 


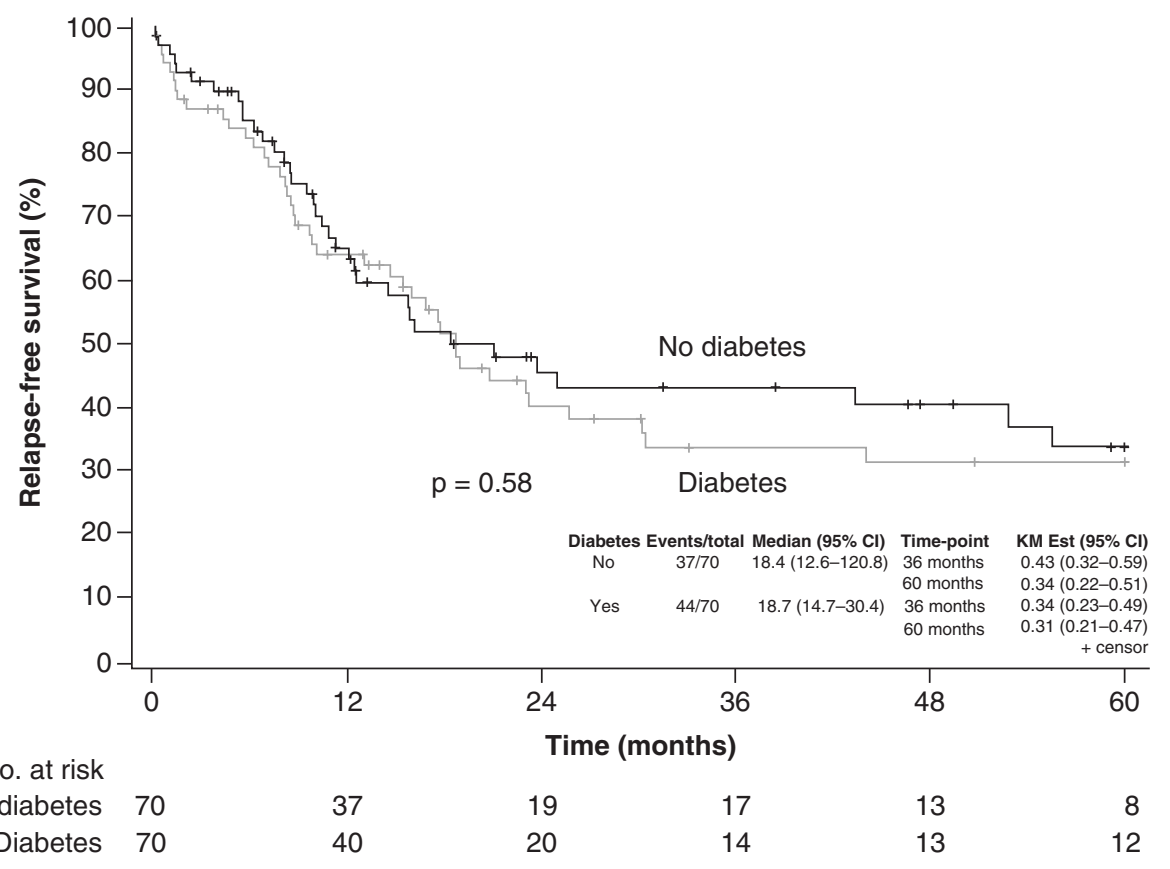

Figure 4. Kaplan-Meier curves estimating relapse-free survival among patients with leukemia. There was no difference in relapse-free survival between patients with and without diabetes $(p=0.58)$.

KM: Kaplan-Meier estimate.

\section{Discussion}

In this retrospective case-control study, diabetes was not associated with a decrease in OS in patients with AML or CML. This should be reassuring to hematologists and endocrinologists who treat patients with AML or CML and diabetes. One possible mechanism for this finding may be the potential protective effects of oral diabetic medications such as metformin, which have shown anticancer properties [18,19]. Interestingly, the majority of diabetes patients were taking oral medication alone for their diabetes. Our data did not distinguish specifically which oral medication was used, but given that metformin is first-line oral therapy for diabetes, it is likely that the vast majority of these patients were taking metformin when these data were collected. In addition, most patients in our study were not taking insulin at the time of leukemia diagnosis. Patients with cancer require close follow-up with their hematologists/oncologists, including regular laboratory measurements that are required to monitor their disease. Therefore, grossly abnormal serum glucose levels can be identified and managed early. Corticosteroids are commonly used in cancer treatment regimens, and early referral to an endocrinologist may be common if a patient's hematologist/oncologist anticipates improved glycemic control before beginning chemotherapy regimens. Another explanation for these findings may be the use of insulin in diabetes patients during leukemia treatment. A recent study in a leukemic mouse model [20] showed that increased levels of insulin-like growth factor binding protein 1 (IGFBP1), an adipokine produced in adipocytes, were associated with insulin resistance and that giving these mice insulin decreased serum IGFBP1 levels.

Leukemia and its treatment also did not appear to affect glycemic control on the basis of $\mathrm{HbA}_{1 \mathrm{c}}$ levels, although the sample size was small. It is unclear why $\mathrm{HbA}_{1 \mathrm{c}}$ levels were not assessed in the majority of diabetes patients with leukemia during the year after leukemia diagnosis; this is an area that should be targeted for quality improvement. Although glucose values increased in patients with diabetes during the year after diagnosis, mean glucose values were not excessively increased among those with diabetes $(<180 \mathrm{mg} / \mathrm{dl})$. Glucose values in this range are most likely acceptable for patients undergoing active treatment for leukemia because intensive glycemic control in patients with diabetes undergoing treatment for leukemia is associated with worse outcomes [21]. Interestingly, patients with diabetes were more likely to be treated with corticosteroids than were controls. The reason for this is unclear, however and this observation should be interpreted cautiously.

We also found higher rates of allelic variations/translocations in diabetes patients with AML and a higher incidence of unfavorable cytogenetics among patients with diabetes. Studies evaluating allelic variation status in 
cancer and diabetes are lacking, particularly in patients with leukemia. One recent study evaluated somatic allelic variations in pancreatic cancer patients with and without diabetes and found a positive association between diabetes duration and somatic variation burden [22]. They also found novel (but infrequent) variations in the diabetes group. Future studies should specifically investigate allelic variational status in patients with leukemia with and without diabetes.

This study had some limitations. The sample size was small, the study duration was short and some relevant data were missing. This study also included a broad study population of patients with AML and CML and patients who had different forms of diabetes. A greedy matching algorithm was used but was limited to age and sex for matching. Other important variables could have been included for matching, but case numbers of patients with diabetes were small. Future studies with larger sample sizes should allow for more granular comparison between these subgroups - a larger prospective study would be ideal. Not all information regarding patient leukemia subtype status was available from the retrospective review. In addition, the majority of the cohort was non-Hispanic, which may limit the applicability to other racial and ethnic groups. A large percentage of patients with diabetes did not have $\mathrm{HbA}_{1 \mathrm{c}}$ values available, because the focus was most likely on cancer treatment. The increase in glucose values also may have resulted from the focus on cancer treatment. Increasing frequency of monitoring of $\mathrm{HbA}_{1 \mathrm{c}}$ values and glucose control represents a potential area of quality improvement.

\section{Conclusion}

In this case-control study, diabetes mellitus was not associated with a decrease in OS in patients with AML/CML. Moreover, AML/CML did not affect glycemic control.

\section{Future perspective}

Despite the study limitations, and similar to findings from most analyses of solid organ cancers, coexisting diabetes was not associated with an increased mortality rate in patients with leukemia. There are limited studies addressing long-term outcomes in patients with leukemia and diabetes; confirmation of these observations in larger patient cohorts is needed. Investigation of the effect of intensive insulin therapy on leukemia survival rate is of interest and worthy of future study.

\section{Summary points}

- In this retrospective case-control study, diabetes was not associated with decreased survival in patients with leukemia (acute myeloid leukemia and chronic myeloid leukemia).

- Hemoglobin $A_{1 c}$ values did not change over time in diabetic patients with leukemia.

- Mean glucose values were significantly different between patients with and without diabetes, but mean glucose levels did not exceed $180 \mathrm{mg} / \mathrm{dl}$.

- Leukemia was not associated with poor glycemic control.

- Among patients with acute myeloid leukemia, the number of allelic variations and/or translocations was significantly higher in those with diabetes.

\section{Author contributions}

All authors conceived the study, collected data, performed the analysis and assisted with the manuscript writing.

\section{Acknowledgments}

Presented virtually at the Annual Meeting of the Endocrine Society, 28-31 March 2020 and published in abstract form: J. Endocr. Soc. 4(Suppl 1), MON-131 (2020).

\section{Financial \& competing interests disclosure}

The authors have no relevant affiliations or financial involvement with any organization or entity with a financial interest in or financial conflict with the subject matter or materials discussed in the manuscript. This includes employment, consultancies, honoraria, stock ownership or options, expert testimony, grants or patents received or pending, or royalties.

No writing assistance was utilized in the production of this manuscript. 
Ethical conduct of research

The authors state that they have obtained appropriate institutional review board approval or have followed the principles outlined in the Declaration of Helsinki for all human or animal experimental investigations. Institutional review board approval was obtained for this retrospective case-control study.

\section{Open access}

This work is licensed under the Creative Commons Attribution 4.0 License. To view a copy of this license, visit http://creativecomm ons.org/licenses/by/4.0/

\section{References}

Papers of special note have been highlighted as: $\bullet$ of interest; $\bullet \bullet$ of considerable interest

1. U.S. Cancer Statistics Working Group. U.S. Cancer Statistics Data Visualizations Tool, based on November 2018 submission data (1999-2016). (2019). www.cdc.gov/cancer/dataviz

2. Centers for Disease Control and Prevention. National Diabetes Statistics Report. (2020). www.cdc.gov/diabetes/data/statistics/statistics-report.html

3. Shi Y, Hu FB. The global implications of diabetes and cancer. Lancet 383(9933), 1947-1948 (2014).

4. Tsilidis KK, Kasimis JC, Lopez DS, Ntzani EE, Ioannidis JP. Type 2 diabetes and cancer: umbrella review of meta-analyses of observational studies. BMJ 350, g7607 (2015).

5. Shanik MH, Xu Y, Skrha J, Dankner R, Zick Y, Roth J. Insulin resistance and hyperinsulinemia: is hyperinsulinemia the cart or the horse? Diabetes Care 31(Suppl. 2), S262-S268 (2008).

6. Hay N. Reprogramming glucose metabolism in cancer: can it be exploited for cancer therapy? Nat. Rev. Cancer 16(10), 635-649 (2016).

7. Gallagher EJ, LeRoith D. The proliferating role of insulin and insulin-like growth factors in cancer. Trends Endocrinol. Metab. 21(10), 610-618 (2010).

8. Deng T, Lyon CJ, Bergin S, Caligiuri MA, Hsueh WA. Obesity, inflammation, and cancer. Annu. Rev. Pathol. 11, 421-449 (2016).

9. Coughlin SS, Calle EE, Teras LR, Petrelli J, Thun MJ. Diabetes mellitus as a predictor of cancer mortality in a large cohort of US adults. Am. J. Epidemiol. 159(12), 1160-1167 (2004).

10. Chen Y, Wu F, Saito E et al. Association between type 2 diabetes and risk of cancer mortality: a pooled analysis of over 771,000 individuals in the Asia Cohort Consortium. Diabetologia 60(6), 1022-1032 (2017).

- Diabetes was associated with an increased risk of death due to several cancers in Asians, with a significant positive association of diabetes and lymphoma.

11. Tawfik B, Pardee TS, Isom S et al. Comorbidity, age, and mortality among adults treated intensively for acute myeloid leukemia (AML). J Geriatr. Oncol. 7(1), 24-31 (2016).

- Diabetes is associated with a decreased 30-day survival in older acute myeloid leukemia patients receiving intensive therapy for acute myeloid leukemia.

12. Karlin N, Verona PM, Buras MR, Kosiorek HE, Cook C. 1297-P: mortality and glycemic control among patients with endometrial cancer and diabetes mellitus: a case-control study. Diabetes 68(Suppl. 1), 1297-P (2019).

13. Karlin NJ, Amin SB, Buras MR, Kosiorek HE, Verona PM, Cook CB. Patient outcomes from lung cancer and diabetes mellitus: a matched case-control study. Future Sci. OA 4(1), FSO248 (2018).

- Diabetes did not decrease survival in patients with lung cancer; lung cancer did not worsen glycemic control.

14. Karlin NJ, Amin SB, Kosiorek HE, Buras MR, Verona PM, Cook CB. Survival and glycemic control outcomes among patients with coexisting pancreatic cancer and diabetes mellitus. Future Sci. OA 4(4), FSO291 (2018).

- Diabetes did not decrease survival in patients with pancreatic cancer.

15. Karlin NJ, Amin SB, Kosiorek HE, Buras MR, Verona PM, Cook CB. Survival and glycemic control in patients with colorectal cancer and diabetes mellitus. Future Sci. OA 4(9), FSO335 (2018).

16. Karlin NJ, Buras MR, Kosiorek HE, Verona PM, Cook CB. Glycemic control and survival of patients with coexisting diabetes mellitus and gastric or esophageal cancer. Future Sci. OA 5(6), FSO397 (2019).

17. Karlin NJ, Mangold AR, Amin SB et al. Survival and glycemic control in patients with coexisting melanoma and diabetes mellitus. Future Sci. OA 5(3), FSO368 (2019).

18. Evans JM, Donnelly LA, Emslie-Smith AM, Alessi DR, Morris AD. Metformin and reduced risk of cancer in diabetic patients. $B M J$ 330(7503), 1304-1305 (2005).

19. Scotland S, Saland E, Skuli N et al. Mitochondrial energetic and AKT status mediate metabolic effects and apoptosis of metformin in human leukemic cells. Leukemia 27(11), 2129-2138 (2013).

-. Metformin leads to reprogramming of intermediary metabolism leading to inhibition of cell proliferation in leukemic cells and apoptosis only in leukemic cells responsive to metformin. 
20. Ye H, Adane B, Khan N et al. Subversion of systemic glucose metabolism as a mechanism to support the growth of leukemia cells. Cancer Cell 34(4), 659-673 e656 (2018).

-. Attenuated leukemia progression and prolonged survival are achieved through disruption of leukemia-induced adaptive homeostasis by inducing high-level production of insulin-like growth factor binding protein 1 from adipose tissue, mediating sensitivity, and leukemia-induced gut dysbiosis, serotonin loss and incretin inactivation, leading to suppression of insulin secretion.

21. Vu K, Busaidy N, Cabanillas ME et al. A randomized controlled trial of an intensive insulin regimen in patients with hyperglycemic acute lymphoblastic leukemia. Clin. Lymphoma Myeloma Leuk. 12(5), 355-362 (2012).

22. Wang K, Zhou W, Meng P et al. Immune-related somatic mutation genes are enriched in PDACs with diabetes. Transl. Oncol. 12(9), 1147-1154 (2019). 